\title{
TuC4
}

\section{Identification of gap soliton through phase measurement}

\author{
Simon-Pierre Gorza, Cyril Cambournac, Philippe Emplit, Marc Haelterman \\ Optique et acoustique, Université libre de Bruxelles CP 194/5; 50, Av. F.D. Roosevelt; B-1050 Bruxelles (Belgium) \\ Tel.: +32-2-650.48.55, Fax: +32-2-650.44.96, Email: sgorza@ulb.ac.be \\ Dirk Taillaert, Bjorn Maes, Roel Baets \\ Department of Information Technology, Ghent University - IMEC; 41, St. Pietersnieuwstraat; B-900o Gent (Belgium)
}

\begin{abstract}
: .
We fully characterize the stationary spatial gap soliton in periodic planar waveguide through the measurement of the transverse phase evolution across the soliton beam.

(C) 2005 Optical Society of America

OCIS codes: (190.3270) Kerr effect, (190.5970) Semiconductor nonlinear optics including MQW, (190.5530)

Pulse propagation and solitons
\end{abstract}

In a 2-D geometry when the refractive index is modulated in one direction $(x)$, the diffraction relation of electromagnetic waves, which relates the longitudinal component of the wave vector $\left(k_{z}\right)$ to the transverse one $\left(k_{x}\right)$ is split up by forbidden bands (photonic band gap). When a Kerr-type nonlinearity is present, the periodic medium supports spatial gap solitons in the form of a bound state of two beams of different propagation angle. These solitons are analogous to temporal gap solitons in 1-D periodic media [1]. Spatial gap solitons can be simply excited by the superposition of two beams (called the forward and backward beam) at the input of a periodic planar waveguide. Therefore, unlike the temporal gap soliton, a stationary gap soliton (with zero transverse velocity with respect to the grating) can be generated. This generation requires two beams of the same power and opposite incident angles both equal to the Bragg angle in such a way that their interference pattern approches the index pattern of the periodic waveguide (see Fig.1.a).

Recently this stationary gap soliton has been observed in semiconductor planar waveguide [2] and in photorefractive crystals [3]. In these experiments the gap soliton has been identified only through the characteristic self-confinement of the constituting beam pair. However a gap soliton is much more that a simple self-confined state akin to standard Kerr-type soliton. Indeed, the gap soliton constitutes a twoparameter family of solutions in which one of the parameters, called detuning, measures the position of the soliton spectrum with respect to the gap of the linear structure. This parameter $\psi$ also determines the phase profile $\phi(x)$ of the gap soliton. This phase profile represents physically the phase difference between the soliton field oscillations (i.e., the beating pattern of the constitutive beams) and the transverse index modulation of the waveguide.

In our work we perform precise measurements of the phase profile $\phi(x)$, which allowed us to determine the value of the detuning parameter $\psi$ of the gap soliton. We provide in this way a complete experimental characterization of the stationary gap soliton.

Our experiment has been performed with laser beams propagating in a periodic planar AlGaAs waveguide that exhibits a self-focusing Kerr nonlinearity at $1.55 \mu \mathrm{m}$. The periodicity is obtained by means of a $4 \mu \mathrm{m}$ grating etched on the top face of the $1.6 \mu \mathrm{m}$-thick guiding layer (see Fig.1.a). Around the Bragg wave number $k_{\mathrm{B}}=\pi / d$ where $d$ is the grating period, the electromagnetic field $\mathcal{E}(x, z)=1 / 2\left(E_{+} \mathrm{e}^{i\left(\beta_{0} z+k_{\mathrm{B}} x\right)}+\right.$ $\left.E_{-} \mathrm{e}^{i\left(\beta_{0} z-k_{\mathrm{B}} x\right)}\right) \mathrm{e}^{-i \omega t}+$ c.c. is ruled by the nonlinear coupled mode equations that describe the evolution of slowly varying envelope of forward $\left(E_{+}\right)$and backward $\left(E_{-}\right)$beams near the Bragg angle $\theta_{\mathrm{B}}=\operatorname{asin}\left(2 \pi / \lambda k_{\mathrm{B}}\right)$ where $\lambda$ is the wavelength in the vacuum [1]:

$$
\begin{aligned}
& \frac{\partial E_{+}}{\partial z}+\frac{k_{\mathrm{B}}}{\beta_{0}} \frac{\partial E_{+}}{\partial x}=i \kappa E_{-}+i \gamma\left(\left|E_{+}\right|^{2}+2\left|E_{-}\right|^{2}\right) E_{+}, \\
& \frac{\partial E_{-}}{\partial z}-\frac{k_{\mathrm{B}}}{\beta_{0}} \frac{\partial E_{-}}{\partial x}=i \kappa E_{+}+i \gamma\left(\left|E_{-}\right|^{2}+2\left|E_{+}\right|^{2}\right) E_{-},
\end{aligned}
$$

where $\kappa$ is the coupling constant that describes the effect of the grating on the propagation and $\gamma$ is the waveguide Kerr nonlinearity coefficient. 


\section{TuC4}

a)

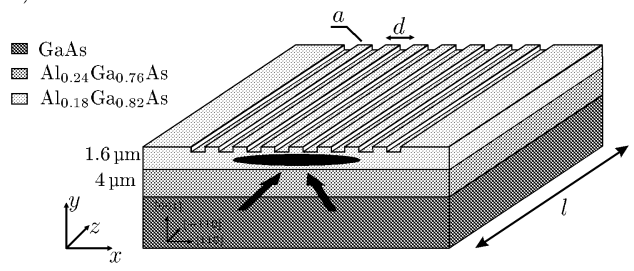

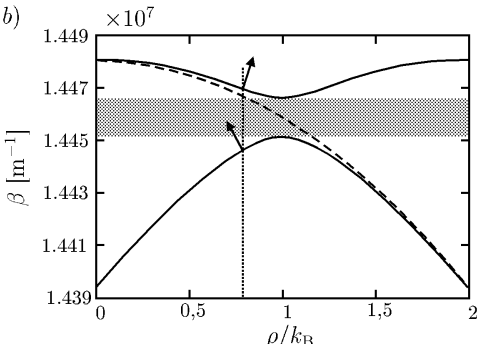

Fig. 1. a) Planar periodic structure: $a=2 \mu \mathrm{m}, \mathrm{d}=4 \mu \mathrm{m}$. b) Band diagram based on Eq. 1 in linear regime.

In the linear propagation regime (i.e. for $\gamma=0$ ) the coupled mode equations show that Bragg reflection on the periodic structure opens a photonic band gap that separates the diffraction relation in two bands. This is shown in the Fig.1.b where we plotted the band structure calculated on the basis of Eq.1 for $\gamma=0$. Around the Bragg angle, this model is equivalent to the Floquet-Bloch analysis for a cosine refractive index modulation. The propagation direction of each mode is given by the normal to the diffraction relation curve (see the arrows in Fig.1b) and the diffraction coefficient is determined by the curvature of this curve. Therefore, when one beam is incident on the structure, it excites modes on the two bands and these modes will travel in opposite direction, as indicated by the black arrows in Fig.1.b [4].
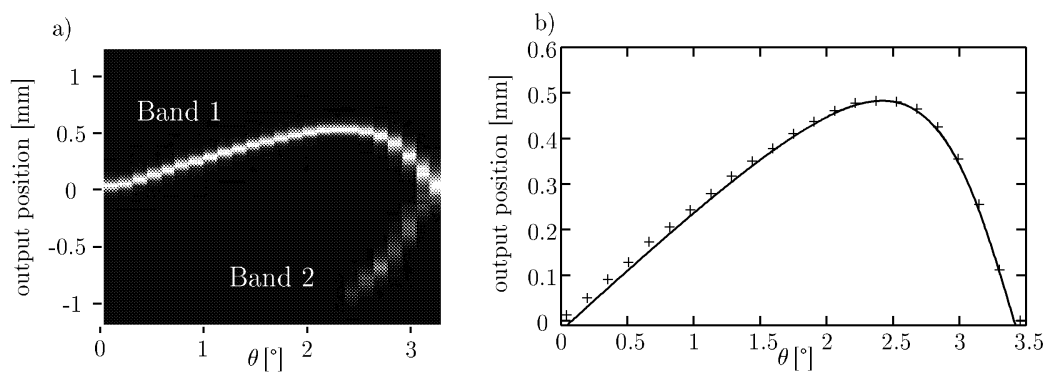

Fig. 2. a) Intensity profile at the output of a $16 \mathrm{~mm}$ long planar periodic waveguide for one incident beam. The angle $\theta$ refers to the input angle in the waveguide. b) Lateral shift at the output for Bloch modes on the first band $(+)$. The solid line shows the theoretical shift based on the diffraction relation plotted on Fig.1.b $\left(\kappa=10300 \mathrm{~m}^{-1}\right)$.

This characteristic feature has been observed at the output of our $16 \mathrm{~mm}$-long sample by launching in the waveguide a single laser beam with a width of $80 \mu \mathrm{m}$ at a wavelength of $1.55 \mu \mathrm{m}$. At this wavelength the Bragg angle is $11.2^{\circ}$ in air $\left(3.4^{\circ}\right.$ in the waveguide). Figure 2.a shows the evolution of the output intensity profile with input angle. We clearly see that for small angles the lateral shift of the beam at the output evolves linearly with the input angle as in an homogeneous medium. On the other hand, near the Bragg angle the propagation is affected by the grating: the propagation angle in the periodic medium decreases as the input angle increases, moreover the input beam excites modes on the first and the second band. At the Bragg angle this two modes travels in the same direction. This allows us, in particular, to identify very accurately the Bragg angle. For Bloch mode on the first band, we plotted the lateral shift at the output as a function of the input angle (see Fig.2.b). Comparison between experimental results and theoretical predictions based on the diffraction relation enables us to deduce the coupling constant $\kappa=10300 \mathrm{~m}^{-1}$ which appears in Eq.1. The knowledge of this value is essential for the measurement of the detuning parameter $\psi$.

When we take into account the nonlinearity $(\gamma \neq 0)$, gap solitons are solutions of Eq.1 [5]. As mentioned above, these solutions are characterized by two parameters $1<\nu<1$ and $0<\psi<\pi$. The first one $(\nu)$ is related to the the soliton transverse velocity, i.e. the angle of propagation of the soliton in the periodic structure. It depends on the relative intensity between the forward and the backward beams and vanishes when these intensities are equals. In that latter case the soliton travels in the $z$ direction and does not move across the index modulation. This is the analogous of the stationary soliton in fiber Bragg gratings. The $\psi$ parameter determines the soliton width, height, and spectrum and plays the role of a detuning parameter. When $\psi \rightarrow 0$ the soliton is wide, the power is low and the spectrum is near the lower branch of the gap. 


\section{TuC4}
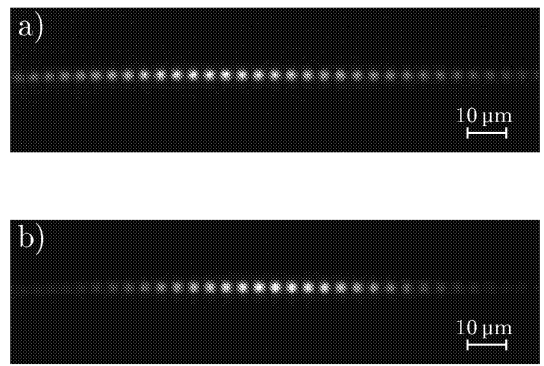

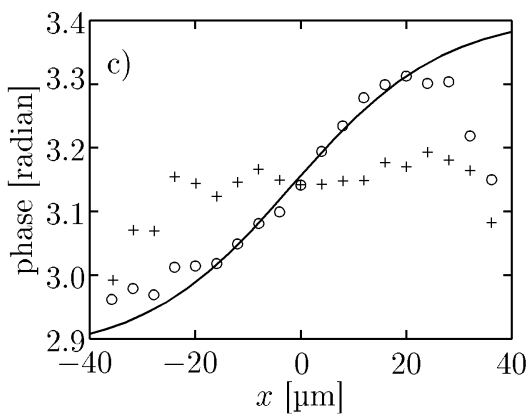

Fig. 3. Output intensity profile at low (a) and high (b) power when a stationary soliton is formed. c) Evolution of the phase of the intensity maxima towards the grating in the case of Fig.a) (+) and Fig. b) (o). The solid line shows the theoretical phase profile for a stationary gap soliton with $\psi=0.27$.

Conversely, a narrow soliton with a spectrum close to the upper branch corresponds to $\psi$ near $\pi$. Moreover as regards the stationary soliton, the periodic pattern is out of phase with respect to the grating in the center of the soliton beam for all power, while in the wings of the beam, the relative phase reaches $\pi \pm \psi$. Therefore we see that even if we cannot measure directly the position of the soliton spectrum in the band gap, the phase evolution across the soliton beam profile allows us to determine the detuning parameter that fully characterizes the stationary gap soliton $(\nu=0)$.

In our experiment, we excite a stationary gap soliton by the superposition of two beams at the Bragg angle as was done in Refs $[2,3]$. The relative phase between these beams is adjusted so that in the linear regime we excite a Bloch mode on the lower branch of the difraction relation. In order to measure the index profile to retrieve the soliton phase profile, we launch in the waveguide a wide beam at normal incidence. At the output, this beam is slightly modulated by the grating and maxima coincide with the index modulation.

Figure 3.a shows the intensity profile at the output of a $4 \mathrm{~mm}$-long periodic waveguide, at low power. Due to the effect of diffraction induced by the grating, the Bloch mode expends up to $100 \mu \mathrm{m}$ FWHM. At high power (see Fig.3.b) the beam focuses in the structure and a stationary gap soliton is formed with a $60 \mu \mathrm{m}$-width. As can be seen on Fig.3.b, the evolution of the phase shift between the intensity pattern and the index modulation is almost flat in the center of the beam, which means that we excite a Bloch mode of the periodic structure. On the other hand, the phase evolves across the soliton profile. This evolution is the one of a gap soliton characterized by a detuning parameter $\psi=0.27$ as shown by the solid line. Moreover the theoretical soliton width for this value of the detuning parameter is $55 \mu \mathrm{m}$ in fully good agreement with the experiment.

In conclusion we fully characterize a stationary gap soliton by measuring its transverse phase profile. A theoretical fit of the measured profile gave us a value of the detuning parameter $\psi$ that is in good agreement with the other soliton parameters such as its width and its intensity.

This work was supported by the Belgian Science Policy Office under grant IAP-V18, the Defay Fund and by the Fonds national de la recherche scientifique (FNRS, Belgium).

\section{References}

[1] J. Feng, "Alternative Scheme for Studying Gap Solitons in an Infinite Periodic Kerr Medium," Opt. Lett. 18(16), 1302 (1993).

[2] D. Mandelik, R. Morandotti, J. S. Aitchison, and Y. Silberberg, "Gap Solitons In Waveguide Arrays," Phys. Rev. Lett. 92, 093,904-1 (2004).

[3] D. Neshev, A. Sukhorukov, B. Hanna, and Y. S. Krolikowski, W. Kivshar, "Controlled Generation and Steering of Spatial Gap Solitons," Phys. Rev. Lett. 93, 083,905 (2004).

[4] D. Mandelik, H. S. Eisenberg, Y. Silberberg, R. Morandotti, and J. S. Aitchison, "Band-Gap Structure of Waveguide Arrays and Excitation of Floquet-Bloch Solitons," Phys. Rev. Lett. 90(5), 053,902 (2003).

[5] C. M. de Sterke and J. E. Sipe, "Gap Soliton," vol. XXXIII of Progress in Optics, p. 203 (1994). 


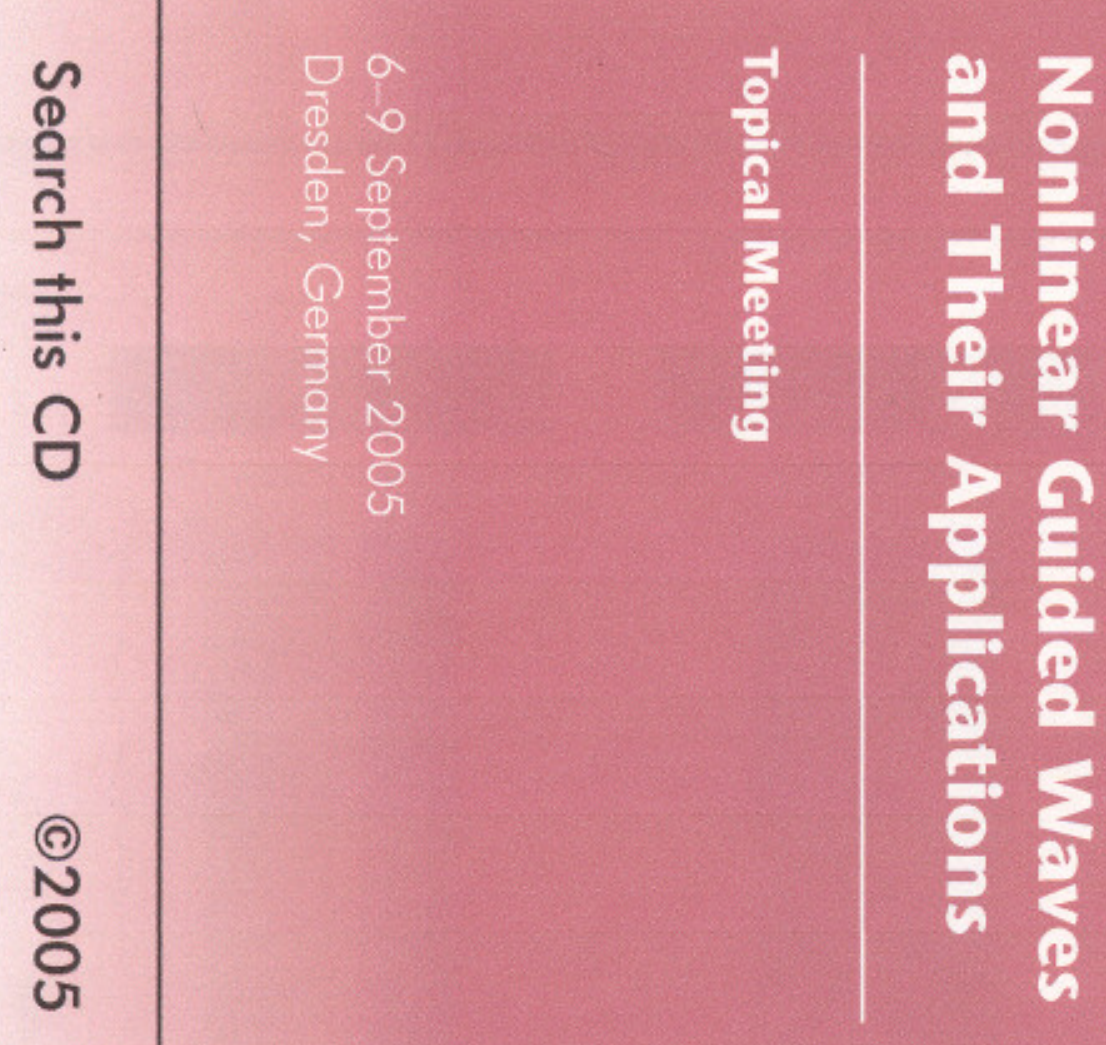

0
0
1
0
0
3
$\frac{1}{D}$
$\frac{1}{0}$

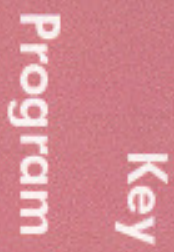

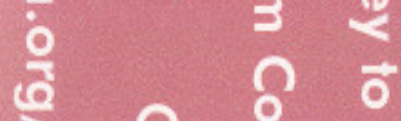

$\xi$
$\vdots$
$\vdots$
$\vdots$
$\vdots$
0
0

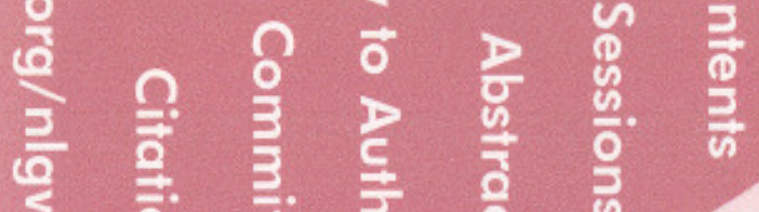

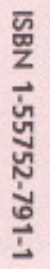

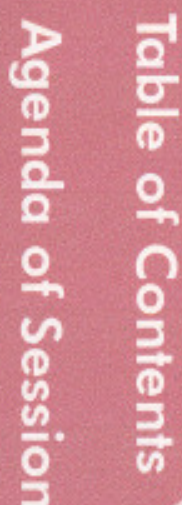

음 क

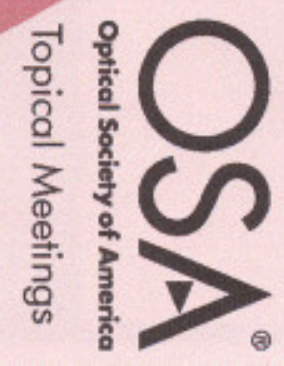

